\title{
A Spectral Factorization Approach to Pseudo-QMF Design
}

\author{
R. David Koilpillai, Member, IEEE, and P. P. Vaidyanathan, Fellow, IEEE
}

\begin{abstract}
A new approach to the design of $M$-channel pseudoquadrature-mirror-filter (QMF) banks is presented. In this approach, the prototype filter is obtained as a spectral factor of a $2 M$ th band filter. This completely eliminates the need for optimization whereas in conventional pseudo-QMF designs, the main computational effort is in optimization of the prototype. As in the conventional approach, the aliasing cancellation (AC) constraint ensures that all the significant aliasing terms are canceled. The overall transfer function $T(z)$ of the analysis/synthesis system has a linear phase and an approximately "flat", magnitude response in the frequency region $\varepsilon \leq \omega \leq(\pi-\varepsilon)$, where $\varepsilon$ depends on the transition bandwidth of the prototype and $0<\varepsilon<(\pi / 2 M)$. Three design examples are included.
\end{abstract}

\section{INTRODUCTION}

$T^{1}$ HE theory of pseudo-quadrature-mirror-filter (QMF) banks and their applications [1]-[6] are well known. The theory deals with the extension of the two-channel QMF solution [7] to $M$ channels, where $M$ is arbitrary. In conventional pseudo-QMF designs [1], [6], we first obtain a linear-phase prototype filter $H(z)$ by optimization. The objective function used in the optimization is a weighted sum of the stopband energy and a "flatness constraint" (which is computed by numerical integration). Once the prototype is obtained, the analysis and synthesis filters are obtained by suitable cosine modulation (incorporating the aliasing cancellation (AC) constraint). So, the main computational effort lies in the optimization of the prototype.

In this paper, we present an approach to pseudo-QMF design that does not involve any optimization. The prototype filter of an $M$ channel filter bank is obtained as a spectral factor of a $2 M$ th band filter. This approach is similar to the conventional pseudo-QMF designs with regard to the derivation of the $\mathrm{AC}$ condition [1], [8], but is different in the way that the filter bank is obtained from the prototype. The main features of the proposed method are:

1) The prototype filter $H(z)$ is obtained, without need for any optimization, by the spectral factorization of $G(z)$, a $2 M$ th band filter. $G(z)$ can be designed by using the standard filter design techniques such as 1) the window-

Manuscript received December 10, 1990; revised December 13, 1991 This work was supported in part by the National Science Foundation under Grants DCI 85 52579, MIP 86 04456, and MIP 89 19196, with matching funds from Hughes Aircraft Company, and Tektronix, Inc.

R. D. Koilpillai is with the Communications Program, G.E. Corporate R\&D Center, Schenectady, NY 12301

P. P. Vaidyanathan is with the Department of Electrical Engineering, California Institute of Technology, Pasadena, CA 91125.

IEEE Log Number 9203331. based filter design [9]; 2) the method in [10], which uses the McClellan-Parks design program [11]; or 3) the eigenfilter approach [12]. Hence, the coefficients of $G(z)$ are readily obtained.

2) As in conventional pseudo-QMF designs, the prototype filter for the new method is designed such that nonadjacent filters of the filter bank do not overlap.

3) The aliasing cancellation (AC) constraint is obtained by using a similar approach as in conventional pseudoQMF designs. The AC constraint ensures that all the significant aliasing terms are canceled.

4) The overall transfer function $T(z)$ of the analysis/ synthesis system has a linear phase. Hence, the QMF circuit is free from phase distortion. However, the prototype filter $H(z)$ does not have a linear phase.

5) The magnitude response $\left|T\left(e^{j \omega}\right)\right|$ is "flat' in the frequency region $\epsilon \leq \omega \leq(\pi-\epsilon)$, where the value of $\epsilon$ depends on the transition bandwidth of the prototype filter, and we always have $0<\epsilon<(\pi / 2 M)$. In this region, the extent of amplitude distortion (i.e., the deviation from flatness) depends on the stopband attenuation of the prototype. The flat response is due to the fact that $H(z)$ is a spectral factor of a $2 M$ th band filter. Consequently, there is no need for a separate flatness constraint. Around $\omega=$ 0 and $\omega=\pi$, the response $\left|T\left(e^{j \omega}\right)\right|$ has dips/bumps. So, except in these frequency regions, the amplitude distortion is small. In speech processing applications, this is acceptable; see for example, [13, chapter 6]. For applications where this is not acceptable (e.g., in image processing) the bumps/dips can be significantly reduced by optimizing a single scalar variable $\theta_{0}$, as demonstrated later in design Example 3.

6) The analysis and synthesis filters are of equal length. We will assume that $(N-1)$, the order of the prototype, is a multiple of $M$ (the number of channels), i.e., ( $N-$ 1) $=m M$, where $m$ is a positive integer. This assumption is made in order to simplify the derivation of the AC constraint.

\section{A. Conventional Pseudo-QMF Designs}

A few salient points of these designs are mentioned here. Please see [1], and [2] for details. Once the prototype filter has been designed, the analysis and synthesis filters are obtained by cosine modulation, as given below:

$$
\begin{gathered}
h_{k}(n)=h(n) \cos \left((2 k+1) \frac{\pi}{2 M}\left(n-\frac{N-1}{2}\right)+\theta_{k}\right), \\
0 \leq k \leq M-1
\end{gathered}
$$




$$
\begin{gathered}
f_{k}(n)=h(n) \cos \left((2 k+1) \frac{\pi}{2 M}\left(n-\frac{N-1}{2}\right)-\theta_{k}\right), \\
0 \leq k \leq M-1
\end{gathered}
$$

where $\theta_{k} \mathrm{~s}$ are chosen such that the significant aliasing terms are canceled [1]. One possible choice is $\theta_{k}=$ $(-1)^{k}(\pi / 4), 0 \leq k \leq M-1$, which yields an overall transfer function $T(z)$ with an approximately "flat" magnitude response (i.e., close to unity gain) at all frequencies. For this choice of $\theta_{k} \mathrm{~s}$, the analysis and synthesis filters do not have linear phase, though the prototype filter has linear phase. Another possible choice is

$$
\theta_{k}= \begin{cases}0, & \text { for } k \text { even } \\ \frac{\pi}{2}, & \text { for } k \text { odd }\end{cases}
$$

In [1], it has been shown that this particular choice of $\theta_{k} \mathrm{~s}$ yields a $T(z)$ with a magnitude response close to unity at all frequencies except around $\omega=0$ and $\omega=\pi$, where it may have a dip or a bump. Such filter banks find use in applications where the regions around $\omega=0$ and $\omega=\pi$ are treated as do not care bands (see [1] and [13, chapter $6])$.

\section{B. Outline of the Paper}

In Section II, the spectral factorization approach to pseudo-QMF bank design is introduced. The modulation by which the analysis and synthesis filters are derived from the prototype filter $H(z)$ is given. Along with that, the relations between the analysis and synthesis filters are stated. Based on this, we get expressions for the channel signals in each of the $M$ branches of the QMF circuit (using approximations to retain only the significant terms). The approximations, which are used, are explained. In Section II-B, we derive the aliasing cancellation (AC) condition that ensures that all the significant aliasing terms are canceled. In Section II-C, the expression for $T(z)$, the overall transfer function of the analysis/synthesis system, is obtained. It is shown that $T(z)$ has an approximately flat response, in the region $\epsilon \leq \omega \leq(\pi-\epsilon)$, due to the fact that $H(z)$ is a spectral factor of a $2 M$ th band filter. The main features of the new approach are also summarized.

Section III deals with the design of the prototype filter, which involves spectral factorization. Three design examples are presented. The Appendix contains a brief description of a spectral factorization approach (based on the inverse LPC technique), which was used in the design examples.

Notations: Boldfaced letters indicate vectors and matrices. Superscripts $T$ and ${ }^{\dagger}$ denote transposition and transposed conjugation, respectively. The tilde accent on a function $\boldsymbol{F}(z)$ is defined such that $\tilde{\boldsymbol{F}}(z)=\boldsymbol{F}_{*}^{T}\left(z^{-1}\right), \forall z$, where the asterisk $\left(^{*}\right)$ subscript denotes the conjugation of coefficients. The complex $W_{k}$ is defined as $W_{k} \triangleq$ $e^{-j(2 \pi / k)}$, for any $k$. If unsubscripted, then $W=W_{M}=$ $e^{-j(2 \pi / M)}$.

\section{Spectral Factorization Approach to QMF DESIGN}

A. The Analysis and Synthesis Filters

Let $H(z)=\Sigma_{n=0}^{N-1} h(n) z^{-n}$ be the prototype filter (with real coefficients). In this approach, since $H(z)$ is obtained by spectral factorization, it does not have linear-phase symmetry. Assume that $(N-1)$, the order of $H(z)$, is a multiple of $M$, the number of channels, i.e., $(N-1)=$ $m M$. (There are no restrictions on $M$ or $m$ ). Let $S_{k}(z)$ be defined as follows:

$$
\begin{aligned}
S_{k}(z) \triangleq & a_{k} H\left(z W_{2 M}^{(k+(1 / 2))}\right) \\
& +a_{k}^{*} H\left(z W_{2 M}^{-(k+(1 / 2))}\right), \quad 0 \leq k \leq M-1
\end{aligned}
$$

where $a_{k} \mathrm{~s}$ are complex constants of unit magnitude; and $W_{2 M}$ is a complex constant as defined in the introduction. $H_{k}(z)$ and $F_{k}(z)$, the analysis and synthesis filter, respectively, of the pseudo-QMF bank are obtained as shown next:

$$
\begin{aligned}
& H_{k}(z)= \begin{cases}S_{k}(z), & \text { for } k \text { even, } \\
z^{-(N-1)} \tilde{S}_{k}(z), & \text { for } k \text { odd },\end{cases} \\
& \quad 0 \leq k \leq M-1
\end{aligned}
$$

As will become evident later, the above choices for the analysis/synthesis filters are essential in the derivation of the AC constraint. Letting $a_{k}=e^{j \theta k}$, we can write (4) (in time domain) as

$$
s_{k}(n)=2 h(n) \cos \left(\frac{\pi}{M}\left(k+\frac{1}{2}\right) n+\theta_{k}\right)
$$

and hence, we get

$$
\begin{aligned}
& h_{k}(n)= \begin{cases}s_{k}(n), & \text { for } k \text { even, } \\
s_{k}(N-1-n), & \text { for } k \text { odd, }\end{cases} \\
& \quad 0 \leq k \leq M-1
\end{aligned}
$$

Next, we define $U_{k}(z)$ and $V_{k}(z)$, which are complexmodulated versions of the prototype $H(z)$. For $0 \leq k \leq$ $M-1$

$$
U_{k}(z) \triangleq H\left(z W_{2 M}^{(k+(1 / 2))}\right), \quad V_{k}(z) \triangleq H\left(z W_{2 M}^{-(k+(1 / 2))}\right) .
$$

Using (10) in (4), $S_{k}(z)$ can be expressed as

$$
S_{k}(z)=a_{k} U_{k}(z)+a_{k}^{*} V_{k}(z), \quad 0 \leq k \leq M-1 .
$$

The signals $Y_{k}(z)$, which are the outputs of the synthesis 


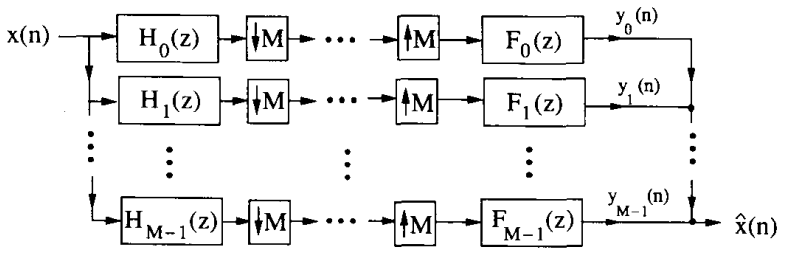

(a)

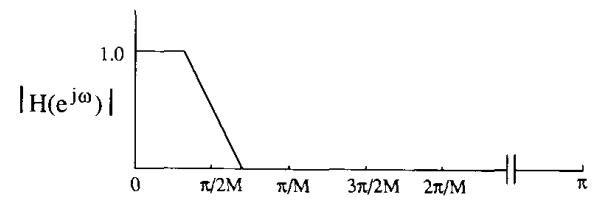

(b)

Fig. 1. (a) The $M$-channel maximally decimated QMF circuit; and (b) the desired response of the prototype $H(z)$.

filters [Fig. 1(a)], are given by

$$
\begin{aligned}
Y_{k}(z)= & \frac{1}{M} F_{k}(z) \sum_{l=0}^{M-1} H_{k}\left(z W^{l}\right) X\left(z W^{l}\right), \\
0 \leq k \leq M-1 & \left\{\begin{array}{l}
\frac{1}{M} F_{k}(z) \sum_{l=0}^{M-1}\left[a_{k} U_{k}\left(z W^{l}\right)\right. \\
\left.+a_{k}^{*} V_{k}\left(z W^{l}\right)\right] X\left(z W^{l}\right), \quad \text { if } k \text { even } \\
\frac{z^{-(N-1)}}{M} F_{k}(z) \sum_{l=0}^{M-1}\left[a_{k}^{*} \tilde{U}_{k}\left(z W^{l}\right)\right. \\
\left.+a_{k} \tilde{V}_{k}\left(z W^{l}\right)\right] X\left(z W^{l}\right), \quad \text { if } k \text { odd. }
\end{array}\right.
\end{aligned}
$$

Note: $(N-1)$ is assumed to be a multiple of $M$.

A key assumption in all pseudo-QMF designs is that filters belonging to nonadjacent channels do not overlap. For example, in a seven-channel pseudo-QMF bank, $\left|H_{3}\left(e^{j \omega}\right)\right|$ has overlap only with $\left|H_{2}\left(e^{j \omega}\right)\right|$ and $\left|H_{4}\left(e^{j \omega}\right)\right|$. The passbands of all the other filters lie in the stopband of $\mathrm{H}_{3}(z)$. In this section, we will repeatedly use this assumption. The following brief discussion will help to clarify the notation and the approximations used in this section. The typical magnitude response of a prototype filter of an $M$-channel pseudo-QMF bank is given in Fig. 1 (b).

Consider for example, the case when $M=4$. In Fig. 2(a) and (b) we have the typical magnitude responses of the analysis and synthesis filters of a four-channel pseudoQMF bank (which satisfies the above assumption). In this figure, $U_{k}(z)$ and $V_{k}(z)$, the modulated versions of the prototype filter, have also been shown. For this case

$$
\begin{gathered}
Y_{k}(z)=\frac{1}{4} F_{k}(z) \sum_{l=0}^{3} H_{k}\left(z W_{4}^{\prime}\right) X\left(z W_{4}^{\prime}\right), \\
0 \leq k \leq 3 .
\end{gathered}
$$

In particular, for $k=2$

$$
Y_{2}(z)=\frac{1}{4} F_{2}(z) \sum_{l=0}^{3}\left[a_{2} U_{2}\left(z W_{4}^{l}\right)+a_{2}^{*} V_{2}\left(z W_{4}^{l}\right)\right] X\left(z W_{4}^{l}\right) \text {. }
$$
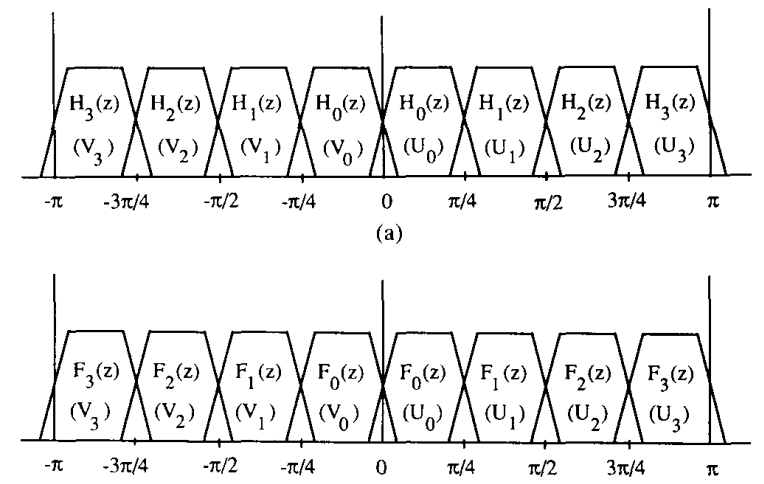

(b)

Fig. 2. A typical four-channel pseudo-QMF bank: (a) the analysis filters $H_{k}(z)$; and (b) the synthesis filters $F_{k}(z)$.
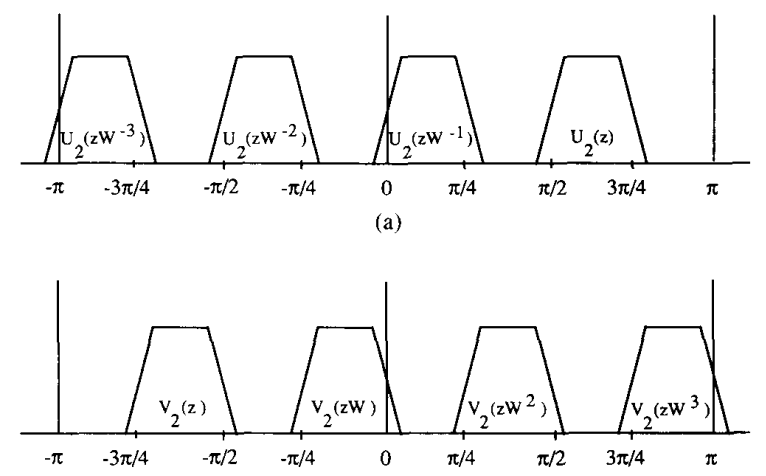

(b)
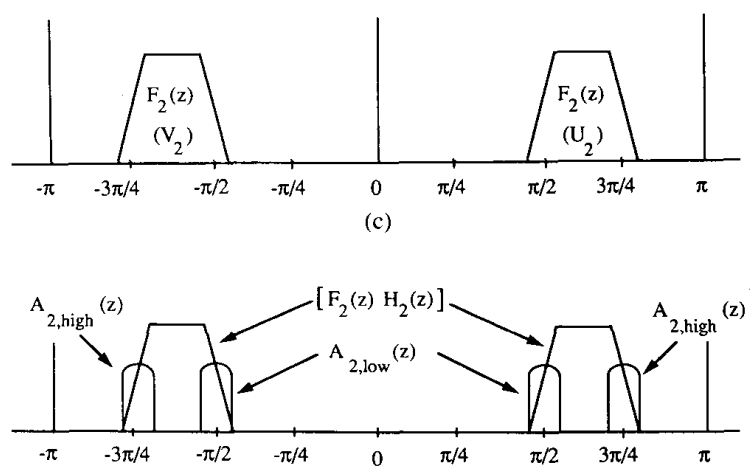

(d)

Fig. 3. The four-channel pseudo-QMF bank $\left(W=W_{4}\right)$ : (a) $U_{2}(z)$ and its shifted versions; (b) $V_{2}(z)$ and its shifted versions; (c) the synthesis filter $F_{2}(z)$; and (d) the six significant terms in $Y_{2}(z)$.

Fig. 3(a) and (b) shows $U_{2}(z), V_{2}(z)$, and their respective frequency shifted versions. In Fig. 3(c) we have the magnitude response of $F_{2}(z)$. From these figures, it can be seen that $U_{2}(z)$ and $V_{2}(z)$ overlap with $F_{2}(z)$. Also, the modulated versions $U_{2}\left(z W_{4}^{-2}\right)$ and $V_{2}\left(z W_{4}^{2}\right)$ overlap with the low-frequency edges of the filter $F_{2}(z)$ while $U_{2}\left(z W_{4}^{-3}\right)$ and $V_{2}\left(z W_{4}^{3}\right)$ overlap with the high-frequency edges of $F_{2}(z)$. So, $Y_{2}(z)$ has a total of six significant 
terms, as shown in Fig. 3(d):

$$
\begin{aligned}
Y_{2}(z)= & \frac{1}{4} F_{2}(z)\left[a_{2} U_{2}(z) X(z)+a_{2}^{*} V_{2}(z) X(z)\right. \\
& +a_{2} U_{2}\left(z W_{4}^{-2}\right) X\left(z W_{4}^{-2}\right) \\
& +a_{2}^{*} V_{2}\left(z W_{4}^{2}\right) X\left(z W_{4}^{2}\right)+a_{2} U_{2}\left(z W_{4}^{-3}\right) X\left(z W_{4}^{-3}\right) \\
& \left.+a_{2}^{*} V_{2}\left(z W_{4}^{3}\right) X\left(z W_{4}^{3}\right)\right] .
\end{aligned}
$$

Using similar reasoning, the expressions in (12) can be simplified as shown next. Neglecting those terms that do not have significant overlap with $F_{k}(z)$, we obtain:

a) For $1 \leq k \leq M-2$ and $k$ even

$$
\begin{aligned}
Y_{k}(z)= & \frac{1}{M} F_{k}(z)\left[a_{k} U_{k}(z) X(z)+a_{k}^{*} V_{k}(z) X(z)\right. \\
& +a_{k} U_{k}\left(z W^{-k}\right) X\left(z W^{-k}\right)+a_{k}^{*} V_{k}\left(z W^{k}\right) X\left(z W^{k}\right) \\
& +a_{k} U_{k}\left(z W^{-(k+1)}\right) X\left(z W^{-(k+1)}\right) \\
& \left.+a_{k}^{*} V_{k}\left(z W^{(k+1)}\right) X\left(z W^{(k+1)}\right)\right] .
\end{aligned}
$$

b) For $1 \leq k \leq M-2$ and $k$ odd

$$
\begin{aligned}
Y_{k}(z)= & \frac{z^{-(N-1)}}{M} F_{k}(z)\left[a_{k}^{*} \tilde{U}_{k}(z) X(z)+a_{k} \tilde{V}_{k}(z) X(z)\right. \\
& +a_{k}^{*} \tilde{U}_{k}\left(z W^{-k}\right) X\left(z W^{-k}\right)+a_{k} \tilde{V}_{k}\left(z W^{k}\right) X\left(z W^{k}\right) \\
& +a_{k}^{*} \tilde{U}_{k}\left(z W^{-(k+1)}\right) X\left(z W^{-(k+1)}\right) \\
& \left.+a_{k} \tilde{V}_{k}\left(z W^{(k+1)}\right) X\left(z W^{(k+1)}\right)\right] .
\end{aligned}
$$

It can be readily verified that for the special case when $M$ $=4$ and $k=2$, (16) reduces to (15). From (16) and (17), we see that the expressions for $Y_{k}(z)$ have four aliasing terms. Of the four terms, two are due to overlap of modulated versions of the input (called images) with the lowfrequency edge of $F_{k}(z)$, and two are due to overlap of images with the high-frequency edge of $F_{k}(z)$. Let them be denoted as $A_{k, \text { low }}(z)$ and $A_{k \text {.high }}(z)$, respectively [as demonstrated in Fig. 3(d)]. For $1 \leq k \leq(M-2)$, we can write:

a) For $k$ even

$$
\begin{aligned}
A_{k, \text { low }}(z) \triangleq & F_{k}(z)\left[a_{k} U_{k}\left(z W^{-k}\right) X\left(z W^{-k}\right)\right. \\
& \left.+a_{k}^{*} V_{k}\left(z W^{k}\right) X\left(z W^{k}\right)\right] \\
A_{k, \text { high }}(z) \triangleq & F_{k}(z)\left[a_{k} U_{k}\left(z W^{-(k+1)}\right) X\left(z W^{-(k+1)}\right)\right. \\
& \left.+a_{k}^{*} V_{k}\left(z W^{(k+1)}\right) X\left(z W^{(k+1)}\right)\right] .
\end{aligned}
$$

b) For $k$ odd

$$
\begin{aligned}
A_{k, \text { low }}(z) \triangleq & z^{-(N-1)} F_{k}(z)\left[a_{k}^{*} \tilde{U}_{k}\left(z W^{-k}\right) X\left(z W^{-k}\right)\right. \\
& \left.+a_{k} \tilde{V}_{k}\left(z W^{k}\right) X\left(z W^{k}\right)\right] \\
A_{k, \text { high }}(z) \triangleq & z^{-(N-1)} F_{k}(z)\left[a_{k}^{*} \tilde{U}_{k}\left(z W^{-(k+1)}\right) X\left(z W^{-(k+1)}\right)\right. \\
& \left.+a_{k} \tilde{V}_{k}\left(z W^{(k+1)}\right) X\left(z W^{(k+1)}\right)\right] .
\end{aligned}
$$

So we can express $Y_{k}(z)$ as

$$
\begin{gathered}
Y_{k}(z)=\frac{1}{M} F_{k}(z) H_{k}(z) X(z)+\frac{1}{M} A_{k, \text { low }}(z)+\frac{1}{M} A_{k, \text { high }}(z), \\
1 \leq k \leq M-2 .
\end{gathered}
$$

$Y_{0}(z)$ has aliasing terms only due to overlap of images with the high-frequency edge of $F_{0}(z)$, which are denoted as $A_{0, \text { high }}(z)$. On the other hand, $Y_{M-1}(z)$ has aliasing terms only due to overlap of images with the low-frequency edge of $F_{M-1}(z)$, which are denoted as $A_{M-1.10 w}(z)$. Hence we get the expressions

$$
\begin{aligned}
Y_{0}(z) & =\frac{1}{M} F_{0}(z) H_{0}(z) X(z)+\frac{1}{M} A_{0, \text { high }}(z) \\
Y_{M-1}(z) & =\frac{1}{M} F_{M-1}(z) H_{M-1}(z) X(z)+\frac{1}{M} A_{M-1, \text { low }}(z) .
\end{aligned}
$$

\section{B. Aliasing Cancellation}

For the new design approach, we will obtain the aliasing cancellation (AC) condition, which ensures that all the significant aliasing terms are canceled, in a manner similar to the conventional pseudo-QMF designs [1]. From the definitions in (18) and (19), we can verify that the magnitude responses of $A_{k, \text { high }}(z)$ and $A_{k+1 \text { low }}(z)$ overlap each other. Hence the condition

$$
A_{k+1 . \text { low }}(z)=-A_{k, \text { high }}(z), \quad 0 \leq k \leq M-2
$$

achieves the cancellation of the aliasing terms between the signals in adjacent channels. If (25) is satisfied, the analysis/synthesis system is said to be "approximately" alias-free, since all the significant aliasing terms have been eliminated. We now derive the conditions under which (25) can be satisfied.

Consider the range $0 \leq k \leq M-2$. Without loss of generality, assume that $k$ is even. The expression for $A_{k, \text { high }}(z)$ is given in (19). Since $(k+1)$ is odd, we get [from (20)]

$$
\begin{aligned}
A_{k+1,10 w}(z)= & z^{-(N-1)} F_{k+1}(z)\left[a_{k+1}^{*} \tilde{U}_{k+1}\left(z W^{-(k+1)}\right)\right. \\
& \cdot X\left(z W^{-(k+1)}\right)+a_{k+1} \tilde{V}_{k+1}\left(z W^{(k+1)}\right) \\
& \left.\cdot X\left(z W^{(k+1)}\right)\right] .
\end{aligned}
$$

Substituting for $A_{k, \text { high }}(z)$ and $A_{k+1, \text { low }}(z)$ in (25), we obtain the following two conditions:

$$
\begin{aligned}
& a_{k} F_{k}(z) U_{k}\left(z W^{-(k+1)}\right) \\
& \quad=-z^{-(N-1)} a_{k+1}^{*} F_{k+1}(z) \tilde{U}_{k+1}\left(z W^{-(k+1)}\right)
\end{aligned}
$$

and

$$
\begin{aligned}
& a_{k}^{*} F_{k}(z) V_{k}\left(z W^{(k+1)}\right) \\
& \quad=-z^{-(N-1)} a_{k+1} F_{k+1}(z) \tilde{V}_{k+1}\left(z W^{(k+1)}\right) .
\end{aligned}
$$


In other words, (25) holds if (27) and (28) are satisfied. Substituting for $F_{k}(z)$, the LHS of (27) becomes

$$
\begin{aligned}
& a_{k} F_{k}(z) U_{k}\left(z W^{-(k+1)}\right) \\
& \quad=z^{-(N-1)} a_{k}\left[a_{k}^{*} \tilde{U}_{k}(z)+a_{k} \tilde{V}_{k}(z)\right] U_{k}\left(z W^{-(k+1)}\right) \\
& \quad=z^{-(N-1)} a_{k}^{2} \tilde{V}_{k}(z) U_{k}\left(z W^{-(k+1)}\right)
\end{aligned}
$$

which is obtained by retaining only the significant term (the omitted term is a product of two terms that do not have significant overlap with each other). In the same manner, substituting for $F_{k+1}(z)$ in the RHS of (27) and simplifying, we get

$$
\begin{aligned}
& z^{-(N-1)} a_{k+1}^{*} F_{k+1}(z) \tilde{U}_{k+1}\left(z W^{-(k+1)}\right) \\
& \quad=z^{-(N-1)} a_{k+1}^{* 2} V_{k+1}(z) \tilde{U}_{k+1}\left(z W^{-(k+1)}\right) .
\end{aligned}
$$

Substituting (30) and (31) in (27), it becomes

$$
\begin{aligned}
& z^{-(N-1)} a_{k}^{2} \tilde{V}_{k}(z) U_{k}\left(z W^{-(k+1)}\right) \\
& \quad=-z^{-(N-1)} a_{k+1}^{* 2} V_{k+1}(z) \tilde{U}_{k+1}\left(z W^{-(k+1)}\right) .
\end{aligned}
$$

From the definitions of $U_{k}(z)$ and $V_{k}(z)$ [from (10)], we have

$$
\tilde{V}_{k}(z)=\tilde{U}_{k+1}\left(z W^{-(k+1)}\right), \quad U_{k}\left(z W^{-(k+1)}\right)=V_{k+1}(z) .
$$

Hence, (32) reduces to

$$
a_{k}^{2}+a_{k+1}^{* 2}=0, \quad 0 \leq k \leq(M-2) .
$$

Choice of $\theta_{k}$ : Two of the several possible choices that satisfy (35) are

$$
\theta_{k}=\frac{\pi}{4}, \quad \forall k
$$

and

$$
\theta_{k}= \begin{cases}0, & \text { if } k \text { is even } \\ \frac{\pi}{2}, & \text { if } k \text { is odd }\end{cases}
$$

\section{The Overall Transfer Function}

With the $\theta_{k}$ chosen to satisfy (35), the analysis/synthesis system is "approximately" alias-free, and the inputoutput equation is given by

$$
\hat{X}(z)=\sum_{k=0}^{M-1} Y_{k}(z) \simeq \frac{1}{M} X(z) \sum_{k=0}^{M-1} F_{k}(z) H_{k}(z) .
$$

Hence, using (6), the overall transfer function $T(z)$ can be expressed as

$$
\frac{\hat{X}(z)}{X(z)} \simeq T(z)=\frac{z^{-(N-1)}}{M} \sum_{k=0}^{M-1} H_{k}(z) \tilde{H}_{k}(z) .
$$

Substituting in (39) from (5) and (11), we get

$$
\begin{aligned}
T(z)= & \frac{z^{-(N-1)}}{M} \sum_{k=0}^{M-1}\left[a_{k} U_{k}(z)+a_{k}^{*} V_{k}(z)\right] \\
& \cdot\left[a_{k}^{*} \tilde{U}_{k}(z)+a_{k} \tilde{V}_{k}(z)\right] .
\end{aligned}
$$

Retaining only the significant terms in (40),

$$
\begin{aligned}
T(z)= & \underbrace{\frac{z^{-(N-1)} \sum_{k=0}^{M-1}\left[U_{k}(z) \tilde{U}_{k}(z)+V_{k}(z) \tilde{V}_{k}(z)\right]}{M}}_{T_{1}(z)} \\
& +\frac{z^{-(N-1)}}{M} \underbrace{\left[a_{0}^{2} U_{0}(z) \tilde{V}_{0}(z)+a_{0}^{* 2} V_{0}(z) \tilde{U}_{0}(z)\right.}_{P_{1}(z)} \\
& +\underbrace{\left.a_{M-1}^{2} U_{M-1}(z) \tilde{V}_{M-1}(z)+a_{M-1}^{* 2} V_{M-1}(z) \tilde{U}_{M-1}(z)\right]}_{P_{2(z)}}
\end{aligned}
$$

The above condition is obtained by starting with (27). It can be verified that if we start with (28), we obtain the same condition as in (34). This result is summarized as follows.

Fact 1: In the proposed approach to pseudo-QMF design, wherein the prototype filter is a spectral factor of a $2 M$ th band filter, with analysis and synthesis filters being obtained by the modulation of the prototype given in (5) and (6), respectively, the condition in (34) is sufficient to ensure that all the significant aliasing terms are canceled. Since $a_{k}=e^{j \theta_{k}}$, the same condition can be expressed in terms of $\theta_{k} \mathrm{~s}$ as

$$
\theta_{k+1}= \pm(2 i+1) \frac{\pi}{2}-\theta_{k}, \quad 0 \leq k \leq M-2
$$

where $i$ is an integer.
Substituting for $U_{k}(z)$ and $V_{k}(z)$ [from (10)], in the expression for $T_{1}(z)$, we obtain

$$
\begin{aligned}
T_{1}(z)= & \frac{z^{-(N-1)}}{M} \sum_{k=0}^{M-1}\left[H\left(z W_{2 M}^{(k+(1 / 2))}\right) \tilde{H}\left(z W_{2 M}^{(k+(1 / 2))}\right)\right. \\
& \left.+H\left(z W_{2 M}^{-(k+(1 / 2))}\right) \tilde{H}\left(z W_{2 M}^{-(k+(1 / 2))}\right)\right] \\
= & \frac{z^{-(N-1)}}{M} \sum_{k=0}^{2 M-1} H\left(z W_{2 M}^{(k+(1 / 2))}\right) \tilde{H}\left(z W_{2 M}^{(k+(1 / 2))}\right) .
\end{aligned}
$$

\section{Flatness Constraint}

In conventional pseudo-QMF systems, we explicitly enforce a flatness constraint in the design of the prototype filter. In the new approach, we will show that if $H(z)$ is 
chosen as a spectral factor of a $2 M$ th band filter, then the transfer function $T(z)$ has an inherent flatness property in the region $\epsilon \leq \omega \leq(\pi-\epsilon)$. This is described below.

Fact 2: Let $G(z)$ be a zero-phase $2 M$ th band filter. Then, as given in [10], $G(z)$ satisfies

$$
\sum_{k=0}^{2 M-1} G\left(z W_{2 M}^{k}\right)=c, \quad \forall z
$$

where $c$ is a constraint.

If the prototype filter $H(z)$ is obtained as a spectral factor of $G(z)$, i.e., satisfying the condition $G(z)=$ $H(z) \tilde{H}(z)$, then we have the following property (by Fact 2):

$$
\sum_{k=0}^{2 M-1} H\left(z W_{2 M}^{k}\right) \tilde{H}\left(z W_{2 M}^{k}\right)=c, \quad \forall z
$$

Using (44) in (42), we obtain

$$
T_{1}(z)=\frac{z^{-(N-1)}}{M} * c
$$

Substituting (45) in (41), we get

$$
T(z)=\frac{z^{-(N-1)}}{M} * c+\frac{z^{-(N-1)}}{M}\left[P_{1}(z)+P_{2}(z)\right]
$$

where $P_{j}(z)$ are the cross terms [defined in (41)] that cannot be eliminated for any choice of $\theta_{k}$. The magnitude response of $P_{1}(z)$ is significant only in the region $|\omega|<$ $\epsilon$, while that of $P_{2}(z)$ is significant only in the region $(\pi$ $-\epsilon)<|\omega|<(\pi+\epsilon)$, where $\epsilon$ depends on the transition bandwidth of $H(z)$ and its value lies in the range $(0$, $(\pi / 2 M))$. As a direct consequence, we see that

$$
\left|T\left(e^{j \omega}\right)\right|=\text { constant }, \quad \epsilon \leq \omega \leq(\pi-\epsilon) .
$$

In the regions around $\omega=0$ and $\omega=\pi$, the response $\left|T\left(e^{j \omega}\right)\right|$ can have bumps/dips, depending on the two cross terms. However, the bumps/dips can be minimized by a suitable choice of the first angle $\theta_{0}$, as demonstrated later in Design Example 3. So the main results of the new approach are:

- The "flat" response, given in (47), is an inherent feature of the proposed design and is due to the fact that the prototype $H(z)$ is a spectral factor of a $2 M$ th band filter.

- No optimization is involved in the design of the prototype filter. $H(z)$. This is the main advantage of this method over conventional pseudo-QMF designs.

- The overall transfer function of the analysis/synthesis system $T(z)$ has linear phase and hence does not have phase distortion. This can be verified from (39) and (46).

\section{E. Comparison with Conventional Pseudo-QMF Banks}

The advantages of the spectral factorization approach are mentioned above. However, it has two disadvantages when compared with the conventional approach. The prototype filter in the conventional designs [1] is a linear phase filter. So, firstly, we can force the overall transfer function to be "flat" at all frequencies. This may be essential in some applications like image processing. Secondly, an efficient implementation of the filter bank based on the discrete cosine transform (DCT) can be derived [2]. In the spectral factorization approach, the prototype does not have linear phase, and hence, does not possess these properties.

On the other hand, in the spectral factorization approach, the flatness constraint is inherently present owing to the $2 M$ th band property of $G(z)$. This result can be utilized in conventional pseudo-QMF designs, as follows. We can force the flatness constraint by including a term in the objective function (for optimization) that makes $H(z) \tilde{H}(z)$ to be as close to a $2 M$ th band filter as possible (i.e., every $2 M$ th impulse response sample from the midpoint must be zero). This is a simple time-domain constraint, which is more readily evaluated than the frequency domain constraint used in [1]. It has been verified that using the above time-domain constraint, the optimization of $H(z)$ converges faster and yields better results.

\section{Design of Prototype Filter}

In the new design approach, the prototype filter $H(z)$ is obtained by the spectral factorization of $G(z)$, a zerophase, $2 M$ th band filter, i.e., $H(z)$ satisfies $G(z)=$ $H(z) \tilde{H}(z)$. Hence, it does not involve any optimization. The order of $H(z)$ is $(N-1)$, i.e., $H(z)=\Sigma_{n=0}^{N-1} h(n) z^{-n}$. The design of $H(z)$ involves the following three steps:

1) Design of $G^{\prime}(z)$ : Let $G^{\prime}(z)=\Sigma_{n=-(N-1)}^{(N-1)} g^{\prime}(n) z^{-n}$, be a zero-phase FIR low-pass filter (noncausal) that is a $2 M$ th band filter, i.e., it satisfies

$$
\sum_{k=0}^{2 M-1} G^{\prime}\left(z W_{2 M}^{k}\right)=\text { constant. }
$$

This condition can be expressed in the time domain as

$$
g^{\prime}(2 p M)= \begin{cases}\frac{1}{2 M}, & p=0, \\ 0, & p \neq 0 .\end{cases}
$$

The filter $G^{\prime}(z)$ (satisfying the above conditions), can be readily designed by the standard window-based filter design techniques [9]. In our design, we will use the Kaiser window [9]. The coefficients of $G^{\prime}(z)$ are obtained as follows:

$$
g^{\prime}(n)=h_{i}(n) w(n), \quad-(N-1) \leq n \leq(N-1)
$$

where $h_{i}(n)$ are the impulse response coefficients of an ideal low-pass filter (with cutoff frequency $=(\pi / 2 M)$ $\mathrm{rad})$, which are given by $h_{i}(n)=(1 / \pi n) \sin ((\pi / 2 M) n)$, and $w(n)$ are the coefficients of a Kaiser window of length $(2 N-1)$. The values of $w(n)$ depend on the value of the window parameter $\beta$ [9]. The main considerations in the choice of $\beta$ are: 
a) If $A_{s}$ is the desired stopband attenuation (in decibels) of $H(z)$, then the stopband attenuation of $G^{\prime}(z)$ must be $\geq\left(2 A_{s}+6\right) \mathrm{dB}$. The additional $6 \mathrm{~dB}$ is to account for the fact that the spectrum will be raised (as explained in the next paragraph) in order to make the spectrum nonnegative (for the spectral factor computation).

b) $G^{\prime}(z)$ should have the same transition bandwidth as $H(z)$.

Having chosen the value of $\beta$, the coefficients of the Kaiser window are easily computed.

2) Design of $G(z)$ : Let $\delta_{2}$ be the stopband ripple of $G^{\prime}(z)$. Then $G(z)$ is obtained as $G(z)=G^{\prime}(z)+\delta_{2}$. For examples in this paper, we use the spectral factorization algorithm presented in the Appendix. For this algorithm, we require that $G(z)$ not have any zeros on the unit circle. So we use $G(z)=G^{\prime}(z)+\delta_{2}+\delta$, where $\delta$ is a positive real constant that "lifts" the zeros of the spectrum lying on the unit circle. $G(z)$ can also be expressed as

$$
\begin{aligned}
& g(0)=g^{\prime}(0)+\delta_{2}+\delta \\
& g(n)=g^{\prime}(n), \quad n \neq 0 .
\end{aligned}
$$

Since $G^{\prime}(z)$ is a $2 M$ th band filter, $G(z)$ is also a $2 M$ th band filter.

3) Design of $H(z)$ by spectral factorization: From its definition in (51), $G\left(e^{j \omega}\right)$ has a real, positive spectrum, i.e., $G\left(e^{j \omega}\right)>0$. Hence, its spectral factor can be computed by the algorithm given in the Appendix or by one of the other spectral factorization methods [18], [19]. Thus, we obtain the desired prototype $H(z)$ satisfying $G(z)$ $=H(z) \tilde{H}(z)$.

Design Example 1: Using the proposed method, a design example for an eight-channel pseudo-QMF bank is presented here. Consider a prototype of length $N=97$. (Its order is 96, which is a multiple of $M$.) First, we obtain the $2 M$ th band filter $G^{\prime}(z)$, whose length $N_{1}=193 . G^{\prime}(z)$ is designed as a Kaiser-window based low-pass filter (LPF) in which the cutoff frequency of the ideal LPF is $\pi / 16$ rads and the Kaiser window parameter $\beta=15.56$. The resultant filter $G^{\prime}(z)$ has stopband attenuation = $147.91 \mathrm{~dB}$ and stopband edge $=0.1138 \pi$ radians. The value of the peak stopband ripple of $G^{\prime}(z)$ is $\delta_{2}=4.023 E$ - 08. Using $\delta=\delta_{2} / 2$ in (51), we obtain the impulse response coefficients of $G(z)$. Hence, the spectrum $G\left(e^{j \omega}\right)$ does not have any zeros on the unit circle.

The prototype filter $H(z)$ is then obtained as a spectral factor of $G(z)$, by using the inverse LPC-based spectral factorization technique (outlined in the Appendix). The resultant prototype $H(z)$ has stopband attenuation $A_{s}=$ $70.94 \mathrm{~dB}$ and the stopband edge $\omega_{s}=0.1138 \pi \mathrm{rad}$. Its magnitude response is shown in Fig. 4(a). The analysis filter bank is obtained by the cosine modulation given in $(5),(8)$ with $\theta_{k}$ chosen as in (36). The responses of all the analysis filters is shown in Fig. 4(b).

For this choice of analysis and synthesis filters $T(z)$, the overall transfer function of the analysis/synthesis system is obtained using (39). Its magnitude response
$\left|T\left(e^{j \omega}\right)\right|$ is plotted in Fig. 4(c), with an expanded view of the "flat" portion shown in (d). For this example, $\left|T\left(e^{j \omega}\right)\right|$ has an approximately flat response in the frequency region $\epsilon \leq \omega \leq(\pi-\epsilon)$, where $\epsilon=0.05 \pi \mathrm{rad}$. In this region, the peak-to-peak error $E_{p-p}=2.288 E-$ $02 \mathrm{~dB}$. From Fig. $4(\mathrm{c})$, it can be seen that $\left|T\left(e^{j \omega}\right)\right|$ has a dip around $\omega=0$ and a bump around $\omega=\pi$. The total aliasing error is defined as $E(\omega) \triangleq(1 / M)\left[\Sigma_{l=1}^{M-1}\right.$ $\left.\left|A_{l}\left(e^{j \omega}\right)\right|^{2}\right]^{1 / 2}$ where $A_{l}(z)=\sum_{k=0}^{M-1} H_{k}\left(z W_{M}^{l}\right) F_{k}(z)$. This error is plotted in Fig. 4(e). Its peak value is $E_{a} \triangleq$ $\max _{\omega} E(\omega)=1.543 E-04$, which confirms that all the significant aliasing terms are indeed canceled.

As mentioned in the Section II-C, the value of $\epsilon$ (and hence, the extent of the region of flat response of $\left.\left|T\left(e^{j \omega}\right)\right|\right)$ depends on the transition bandwidth of the prototype filter. On the other hand, the errors $E_{p-p}$ and $E_{a}$ depend on the $A_{s}$ of the prototype (i.e., the higher the stopband attenuation, the lower the values of $E_{p-p}$ and $E_{a}$ ). So for a given length of the prototype filter, the tradeoff between the transition bandwidth and $A_{s}$ is reflected as a tradeoff (for the overall analysis/synthesis system) between $\epsilon$ and the errors $E_{p-p}, E_{a}$. To illustrate this fact, we present another design example.

Design Example 2: This is also an eight-channel pseudo-QMF bank designed in an identical manner to the previous example, using the same filter length, but the prototype filter in this example has a narrower transition bandwidth and lower stopband attenuation (when compared to the prototype of Design Example 1). $G^{\prime}(z)$, whose length $N_{1}=193$, is designed by using a Kaiser widow parameter $\beta=10.5$. It has stopband attenuation $=104.19 \mathrm{~dB}$ and stopband edge $=0.0976 \pi \mathrm{rad}$. The value of $\delta_{2}=6.174 E-06$ and the impulse response coefficients of $G(z)$ are obtained by using $\delta=\delta_{2} / 2$ in (51). Using the same spectral factorization algorithm, we obtain the prototype $H(z)$, which has $A_{s}=48.82 \mathrm{~dB}$ and $\omega_{s}$ $=0.0976 \pi$ rad. Its magnitude response is shown in Fig. $5(a)$.

Comparing this prototype with that of the previous example, the tradeoff between the transition bandwidth and $A_{s}$ is evident. The responses of all the analysis/synthesis filters is shown in Fig. 5(b). The magnitude response $\left|T\left(e^{j \omega}\right)\right|$ is plotted in Fig. 5(c), and (d), and the total aliasing error is given in (e). For this example, $\epsilon=0.0348 \pi$ rad, while $E_{p-p}=0.1407 \mathrm{~dB}$ and $E_{a}=2.77 E-03$. So, in this example $\left|T\left(e^{j \omega}\right)\right|$ has an approximately flat response over a wider region than in the previous example, but the errors $E_{p-p}, E_{a}$ are noticeably bigger. In general, it has been observed that, in the spectral factorization approach to pseudo-QMF design, choosing the prototype with the higher stopband attenuation yields a better pseudo-QMF design.

Design Example 3: The main purpose of this example is to show how the dips/bumps in the magnitude response of the overall transfer function $T(z)$ around $\omega=0$ and $\omega$ $=\pi$, can be minimized. This is particularly useful in image processing applications. The design parameters are the same as the ones in Design Example 2. The main dif- 


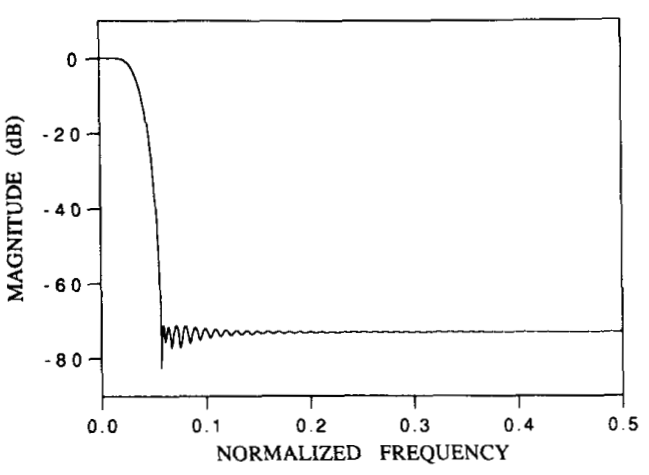

(a)

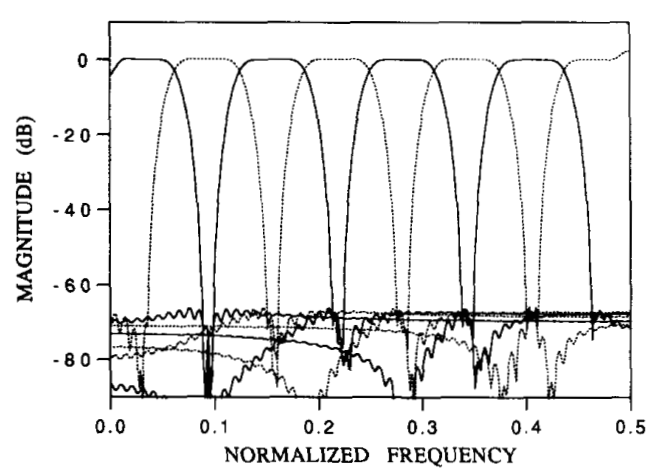

(b)

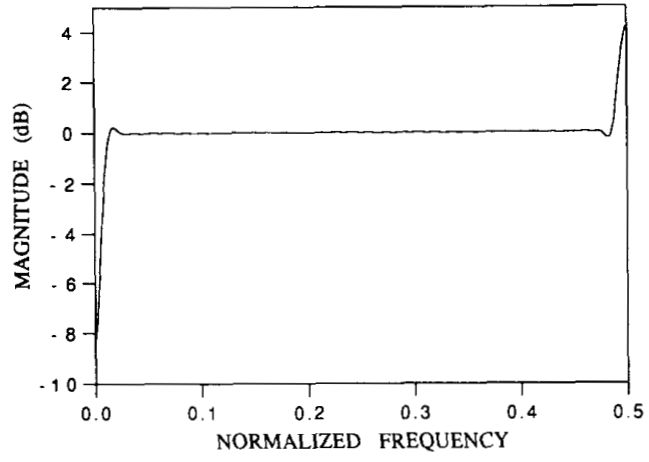

(c)

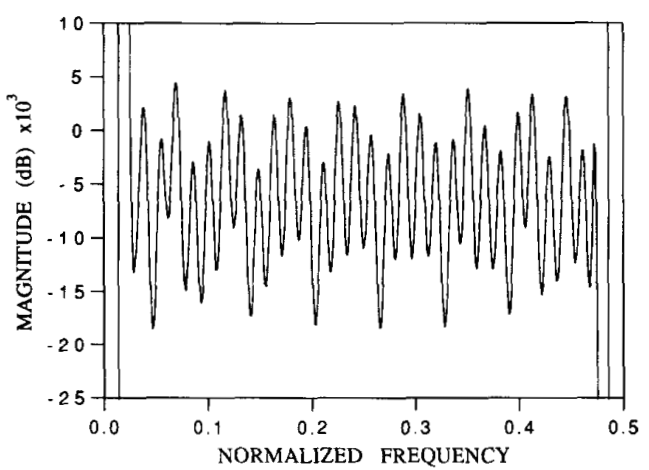

(d)

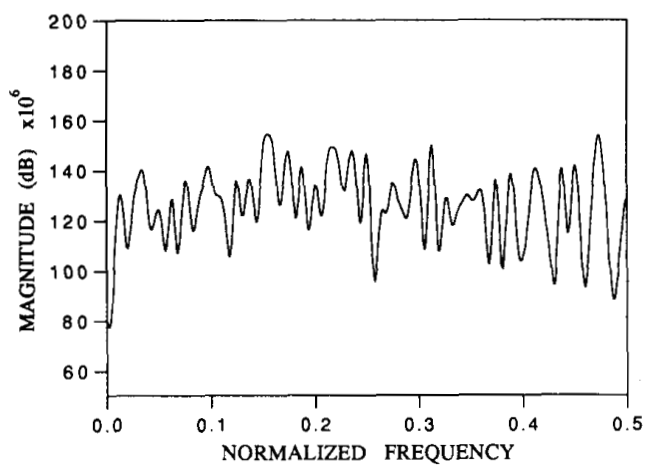

(e)

Fig. 4. Design Example 1: (a) response of the prototype filter $H(z)$; (b) response of the eight-channel filter bank; (c) magnitude response of $T(z)$; (d) expanded view of $(c)$; and (e) plot of aliasing error.

ference is in the choice of $\theta_{k}$ used to obtain the analysis and synthesis filters. In Design Example 2, they were chosen as $\theta_{k}=\pi / 4, \forall k$. In this example, we will optimize $\theta_{0}$ such that the distortion in $T(z)$ around $\omega=0$ and $\omega=\pi$ is minimized, and then the other $\theta \mathrm{s}$, as obtained using the relation given in (35), are

$$
\theta_{k+1}=\frac{\pi}{2}-\theta_{k}, \quad 1 \leq k \leq M-2 .
$$

This is a single-parameter optimization, and can be done using any standard technique. For this example, the best value was found to be $\theta_{0}=0.2121 \mathrm{rad}$. Fig. 6(a) shows the magnitude response of the analysis filter bank. Fig. 6(b) shows the magnitude response of $T(z)$. Comparing Fig. 6(b) with the plot of Fig. 5(c), we note the significant improvement around $\omega=0$ and $\omega=\pi$. As in the other examples, the residual aliasing error is negligible.

This example serves to verify that a suitable choice of 


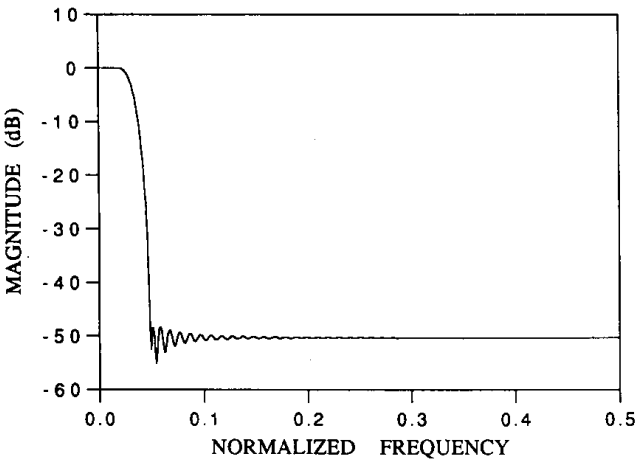

(a)

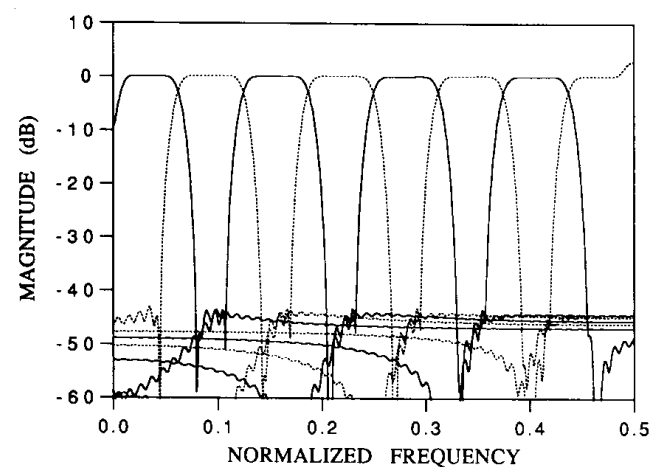

(b)

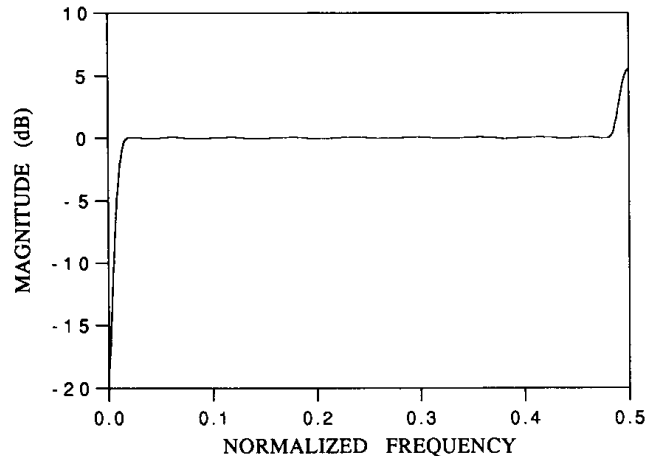

(c)

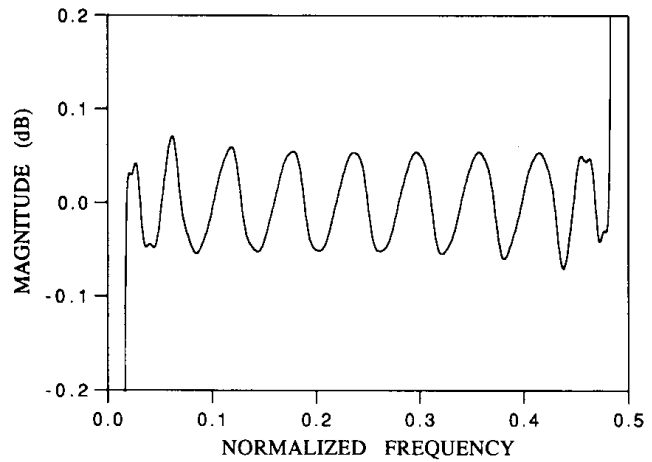

(d)

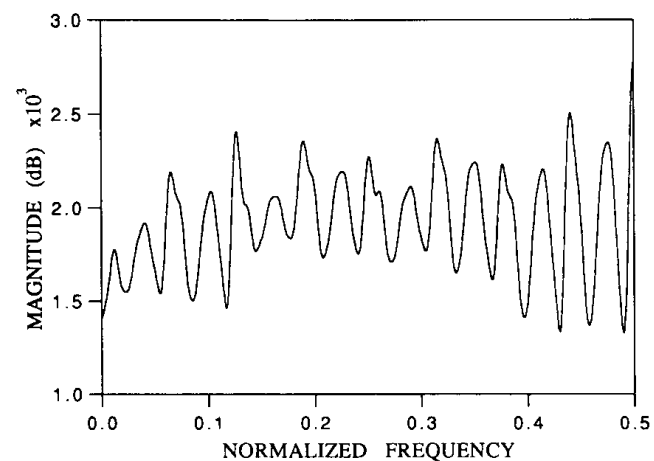

(e)

Fig. 5. Design Example 2: (a) response of the prototype filter $H(z)$; (b) response of the eight-channel filter bank; (c) magnitude response of $T(z)$; (d) expanded view of (c); and (e) plot of aliasing error.

$\theta_{0}$ can significantly improve the flatness of $T(z)$ around $\omega$ $=0$ and $\omega=\pi$.

\section{Summary}

A new approach to pseudo-QMF design, based on spectral factorization, is presented. The main advantage of this approach over conventional pseudo-QMF designs is that no optimization is involved in the design of the prototype. The aliasing cancellation (AC) constraint en- sures that all the significant aliasing terms are canceled. The overall transfer function of the analysis/synthesis system has a "flat" response in the frequency region $\epsilon \leq$ $\omega \leq(\pi-\epsilon)$, where $\epsilon$ depends on the transition bandwidth of the prototype filter and $0<\epsilon<\pi / 2 M$. Design examples of pseudo-QMF banks, designed by the spectral factorization approach, are included. Furthermore by optimizing a single parameter (i.e., $\theta_{0}$ ), it is possible to reduce the amplitude distortion around $\omega=0$ and $\pi$. 


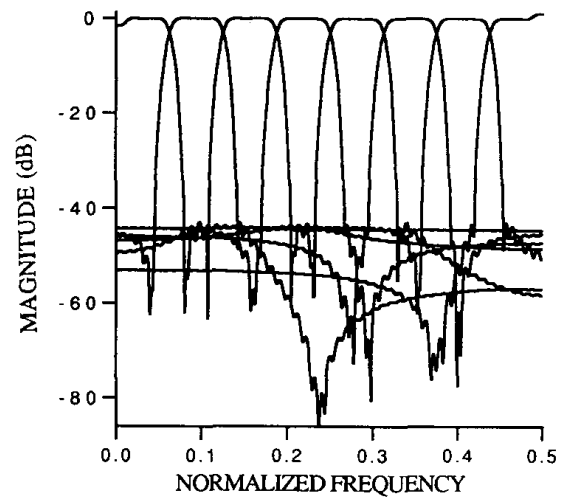

(a)

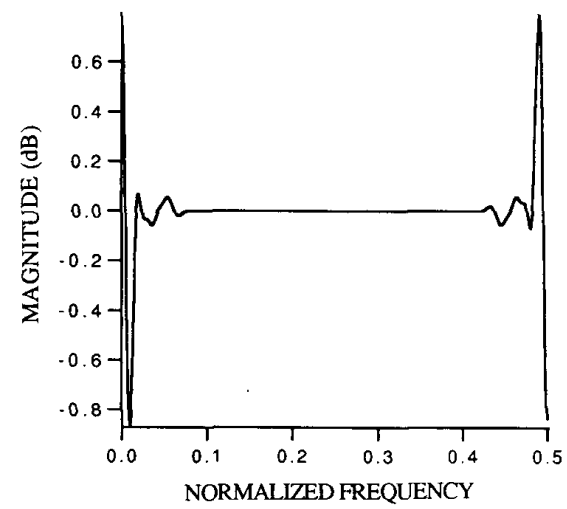

(b)

Fig. 6. Design Example 3: (a) response of the eight-channel filter bank; and (b) magnitude response of $T(z)$.

APPENDIX

Spectral Factorization Using the Inverse LPC TECHNIQUE

In this Appendix, we present an algorithm for spectral factorization. This efficient, noniterative algorithm is based on the inverse linear predictive coding (LPC) technique and can be used to compute the minimum phase spectral factor of any moving average (MA) autocorrelation sequence.

The MA process is one of the basic models of timeseries analysis and stochastic modeling of linear systems. In a variety of situations, we are interested in finding a spectral factor of an MA power spectrum. The spectral factorization problem can be stated as follows: given a real MA autocorrelation sequence $r(k)$, satisfying $r(k)=$ $0,|k|>N$ and $r(-k)=r(k) . S(z)$, the Z-transform of $r(k)$, is given by $S(z)=\Sigma_{k=-N}^{N} r(k) z^{-k}$, and $S\left(e^{j \omega}\right)$ is the power spectrum of $r(k)$. Spectral factorization involves the computation of a polynomial $C(z)=\sum_{n=0}^{N} c(n) z^{-n}$, such that it satisfies $S(z)=C(z) C\left(z^{-1}\right)$. In order to obtain a unique solution for $C(z)$, we impose the constraint that $C(z)$ should be minimum phase (i.e., none of the zeros are outside the unit circle in the $Z$-plane).

In parametric modeling [14], [15], given the autocorrelation sequence, the parameters of the autoregressive
(AR) model can be determined by solving the YuleWalker equations (which are linear). On the other hand, computing the MA model parameters involves nonlinear equations, and so, an indirect approach is to use AR modeling to do the desired computation. One such method is given in [16], where the MA parameters are obtained by evaluating the $\mathrm{AR}$ model parameters corresponding to the inverse autocorrelations. This is also known as the "inverse LPC technique'" [17].

The spectral factorization algorithm that we present is a "one-pass", algorithm (noniterative) based on the inverse LPC technique. The underlying theory is simple. This algorithm works well, except if the zeros of $S(z)$ are on the unit circle, and in this particular case, we have a modified approach. This algorithm is comparable with the other iterative and noniterative algorithms [18], [19], [20] in terms of the accuracy of the spectral factor.

\section{A. The Spectral Factorization Algorithm}

This method of computing the minimum phase spectral factor involves the following steps:

1) Compute the samples of the power spectrum $S\left(e^{j \omega}\right)$ by evaluating the DFT (using FFT) of the MA autocorrelation sequence $r(n)$.

$$
\begin{gathered}
\left.R(k) \triangleq S\left(e^{j \omega}\right)\right|_{\omega=(2 \pi / M) k}=\sum_{n=-N}^{N} r(n) W_{M}^{n k}, \\
0 \leq k \leq M-1
\end{gathered}
$$

where $W_{M}=e^{-j 2 \pi / M} \cdot M$, the number of FFT points, is typically chosen to be much larger than $(2 N+1)$, the length of the autocorrelation sequence [as justified in step 3].

2) Obtain the FFT samples of the inverse power spectrum.

$$
\begin{aligned}
\left.S_{\mathrm{inv}}\left(e^{j \omega}\right)\right|_{\omega=(2 \pi / M) k}=R_{\mathrm{inv}}(k) & \triangleq \frac{1}{R(k)}, \\
0 & \leq k \leq M-1
\end{aligned}
$$

where the inverse power spectrum $S_{\mathrm{inv}}\left(e^{j \omega}\right)$ is defined as the reciprocal of the original power spectrum $S\left(e^{j \omega}\right)$, assuming that $S(z)$ does not have any zeros on the unit circle.

3) Obtain $r_{\text {inv }}(n)$, the inverse autocorrelation sequence (i.e., the autocorrelation sequence associated with the inverse power spectrum), as given by $r_{\text {inv }}(n)=$ IFFT $\left[R_{\text {inv }}(k)\right]$. Typically, $r_{\text {inv }}(n)$ is a doubly infinite sequence. In order to avoid time-domain aliasing (which is an artifact of FFT), we choose $M \gg(2 N+1)$.

4) Having obtained $r_{\text {inv }}(n)$, we use the Levinson-Durbin recursion to compute $C(z)$, the desired spectral factor. $C(z)$ satisfies the equation

$$
S_{\text {inv }}\left(e^{j \omega}\right)=\frac{1}{S\left(e^{j \omega}\right)}=\frac{1}{\left|C\left(e^{j \omega}\right)\right|^{2}} .
$$

Therefore, $S(z)=C(z) C\left(z^{-1}\right)$, and $C(z)$ is guaranteed to be minimum phase. 
Note: The above 'method works well even if the zeros of the power spectrum are close to the unit circle. But if there are zeros on the unit circle, the spectrum must be "raised" by adding a positive constant $\delta$, i.e., $S^{\prime}\left(e^{j \omega}\right)=$ $S\left(e^{j \omega}\right)+\delta$. However, the value of $\delta$ can be chosen to be small enough such that the computed spectral factor [of $\left.S^{\prime}(z)\right]$ is very close to the spectral factor of $S(z)$.

\section{B. Computational Complexity}

Using the inverse LPC method, the total computation involved are two $M$-point FFT's, the reciprocation of the FFT samples $S(k)$ and a Levinson-Durbin recursion of $\operatorname{order} N$. As the value of $M$ is increased, the accuracy of the spectral factor also increases. The spectral factorization algorithm works well for most cases, even when the order of the spectral factor is high and the zeros of the spectrum are close to the unit circle.

\section{ACKNOWLEDGMENT}

The authors are indebted to I. Djokovic for suggesting the optimization of $\theta_{0}$, and for generously supplying Design Example 3.

\section{REFERENCES}

[1] J. H. Rothweiler, "Polyphase quadrature filters-a new subband coding technique," in Proc. IEEE Int. Conf. Acoust., Speech. Signal Processing, Boston, 1983, pp. 1280-1283.

[2] J. Masson and Z. Picel, "Flexible design of computationally efficient nearly perfect QMF filter banks," in Proc. IEEE Trans. Acoust., Speech, Signal Processing, Tampa, FL, Mar. 1985, pp. 14.7.114.7.4.

[3] H. J. Nussbaumer, "Pseudo QMF filter bank," IBM Tech. Disclosure Bull., vol. 24, no. 6, pp. 3081-3087, Nov. 1981.

[4] H. J. Nussbaumer and M. Vetterli, "Computationally efficient QMF filter banks," in IEEE Trans. Acoust., Speech. Signal Processing. San Diego, CA. Mar. 1984,pp. 11.3.1-11.3.4

[5] P. L. Chu, "Quadrature mirror filter design for an arbitrary number of equal bandwidth channels," IEEE Trans. Acoust., Speech. Signal Processing, vol. ASSP-33, no. 1, pp. 203-218, Feb. 1985.

[6] R. V. Cox, "The design of uniformly and nonuniformly spaced pseudoquadrature mirror filters," IEEE Trans. Acoust., Speech, Signal Processing, vol. ASSP-34, no. 5, pp. 1090-1096, Oct. 1986.

[7] A. Croisier, D. Esteban, and C. Galand, "Perfect channel splitting by use of interpolation/decimation/tree decomposition techniques," in Proc. Int. Conf. Informat. Sci. Syst., Patras, Greece, 1976, pp. 443-446.

[8] R. D. Koilpillai, T. Q. Nguyen, and P. P. Vaidyanathan, "Some results in the theory of crosstalk-free transmultiplexers." IEEE Trans. Signal Processing, vol. 39, pp. 2174-2183, Oct. 1991.

[9] P. P. Vaidyanathan, "Design and implementation of digital FIR filters," in Handbook of Digital Signal Processing, D. F. Elliot, Ed. New York: Academic, 1987, chapter 2.

[10] F. Mintzer, "On half-band, third-band, and Nth-band FIR filters and their design," IEEE Trans. Acoust., Speech, Signal Processing, vol. ASSP-30, pp. 734-738, Oct. 1982.

[11] J. H. McClellan and T. W. Parks, "A unified approach to the design of optimum FIR linear-phase digital filters," IEEE Trans. Circuit Theory, vol. CT-20, pp. 697-701, Nov. 1973.

[12] P. P. Vaidyanathan and T. Q. Nguyen, "Eigenfilters: A new approach to least squares FIR filter design and applications including Nyquist filters,"'IEEE Trans. Circuits Syst., vol. CAS-34, no. 1, pp. 11-23, Jan. 1987.
[13] L. R. Rabiner and R. W. Schafer, Digital Processing of Speech Signals. Englewood Cliffs. NJ: Prentice-Hall, 1978.

[14] L. B. Jackson, Digital Filters and Signal Processing. Boston, MA Kluwer Academic Publishers, 1986.

[15] S. M. Kay, Modern Spectral Estimation: Theory and Applications Englewood Cliffs, NJ: Prentice-Hall, 1988.

[16] W. S. Cleveland, "The inverse autocorrelations of a time series and their applications," Technometrics, vol. 14, no. 2, pp. 277-293, May 1972 .

[17] C. E. Schmid, "Design of IIR/FIR filters using a frequency domain bootstrapping technique and LPC methods," IEEE Trans. Acoust. Speech, Signal Processing, vol. ASSP-31, no. 4, pp. 999-1006, Aug. 1983.

[18] G. A. Mian and A. P. Nainer, "A fast procedure to design equiripple minimum phase FIR filters," IEEE Trans. Circuits Syst., pp. 327331, May 1982.

[19] B. Friedlander, "A lattice algorithm for factoring the spectrum of a moving average process," IEEE Trans. Automat. Contr., vol. AC-28, no. 11 , pp. 1051-1055, Nov. 1983.

[20] C. T. Mullis and C. J. Demeure, "The Jury matrix and a NewtonRaphson procedure for moving average spectral factorization," in Proc. Asilomar Conf. Signals, Syst., Comput., 1987, pp. 635-639.

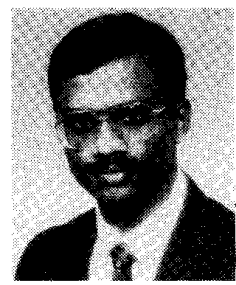

R. David Koilpillai (S'83-M'90) was born in Madras, India, on February 18, 1962. He received the $B$. Tech. degree from the Indian Institute of Technology, Madras, in 1984 and the M.S. and $\mathrm{Ph} . \mathrm{D}$. degrees from the California Institute of Technology, in 1985 and 1991 respectively, all in electrical engineering.

$\mathrm{He}$ is currently on the staff of the Communications Program at the General Electric Corporate R\&D Center in Schenectady, NY. His main research interests are in the areas of DSP applications in communications, multirate systems, adaptive equalization, and theory and design of digital filter banks.

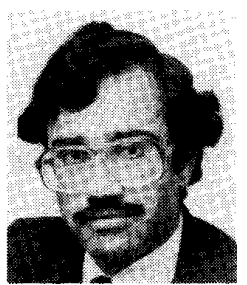

P. P. Vaidyanathan (S'80-M'83-SM'88-F'91) was born in Calcutta, India, on October 16, 1954 $\mathrm{He}$ received the $\mathrm{B}$.Sc. (Hons.) degree in physics and the B.Tech. and M.Tech. degrees in radiophysics and electronics, all from the University of Calcutta, India, in 1974, 1977, and 1979, respectively, and the Ph.D. degree in electrical and computer engineering from the University of California, Santa Barbara, in 1982.

He was a postdoctoral fellow at the University of California, Santa Barbara, from September 1982 to March 1983. In March 1983 he joined the Electrical Engineering Department of the California Institute of Technology as an Assistant Professor, and since 1988 has been an Associate Professor of electrical engineering there. His main research interests are in digital signal processing, multirate systems, wavelet transforms, and adaptive filtering.

Dr. Vaidyanathan served as Vice-Chairman of the Technical Program Committee for the 1983 IEEE International Symposium on Circuits and Systems, and as an Associate Editor for the IEEE TransaCtIons ON CIRCUITS AND SYSTEMS for the period of 1985-1987. He also served as the Technical Program Chairman for the 1992 IEEE International Symposium on Circuits and Systems. He was a recipient of the Award for Excellence in Teaching at the California Institute of Technology for the year 19831984. He also received the NSF's Presidential Young Investigator award in 1986. In 1989 he received the IEEE ASSP Senior Award for his paper on multirate perfect-reconstruction filter banks. In 1990 he was recipient of the S. K. Mitra Memorial Award from the Institute of Electronics and Telecommunications Engineers, India, for his joint paper in the IETE journal. He was elected Fellow of the IEEE in 1991. 